\title{
The Women's Mental Health Conference at Yale: Trainees Lead the Way to Improving Care for Women
}

\author{
Stefanie Gillson ${ }^{1} \cdot$ Sofia Noori ${ }^{1}$
}

Received: 15 February 2020 / Accepted: 11 August 2020 / Published online: 28 August 2020

(C) Academic Psychiatry 2020

To the Editor:

On October 25, 2019, over 400 people gathered at the Yale School of Medicine to attend the nation's first trainee-led multidisciplinary Women's Mental Health Conference. The audience was composed of advocates, researchers, clinicians, and community members from across the nation and world. The conference commenced with a keynote by V (formerly Eve Ensler), recognized globally for her advocacy to end gender-based violence and as the author of The Vagina Monologues. The conference was free and open to the public.

Following V's rousing keynote, attendees separated into small groups. They participated in learning sessions created by a wide range of women's mental health professionals, who shared their personal experiences and expertise on topics such as human trafficking, perinatal mental health, and eating disorders. The conference also touched upon pressing contemporary issues in women's mental health, including the effect of colorism on women of color, transwomen's mental health, and the intersections of culture, race, and gender.

During the preceding year, a planning committee of 20 graduate students (from the schools of medical, public health, and law), undergraduate students, and resident physicians (from the departments of psychiatry, obstetrics, and gynecology) organized the conference to increase awareness and training on the wide range of unique mental health issues affecting women. The overarching goals of the conference were to create a community across multiple disciplines to discuss ways of collaboration to improve women's mental health, provide attendees with practical skills, and highlight the contributions of women's mental health advocates, researchers, and clinicians.

Stefanie Gillson

stefanie.gillson@yale.edu

Yale School of Medicine, New Haven, CT, USA
The success of the conference exceeded all expectations and can be attributed to the dedication of the multidisciplinary committee and their advisors. The members were impassioned and incredibly mission driven; they devoted countless hours throughout the year preceding the conference to fundraising, planning, and inviting speakers. Impressively, the all-trainee committee raised over $\$ 20,000$ to make the conference completely free. Following the conference, attendees received a feedback survey that revealed nearly universally positive feedback. Many commented that the keynote address was the highlight of the conference and that they appreciated the depth and variety of the presenters. Of those who took the survey, $100 \%$ of the respondents rated their experience at the conference as either excellent $(76 \%$, the highest score of a 5-point rating scale) or very good ( $24 \%$, the second-highest score).

Our committee has been amazed by the overwhelming reception to the inaugural conference, which has spurred the organizers to design a bigger vision for future iterations. Given the success of the 2019 event, the organizers are actively fundraising to continue the conference for the foreseeable future.

With the rise of \#MeToo and other grassroots movements, recognition of women's mental health and the many factors that influence it is increasing, within both public and academic spheres.

The scarcity of women's mental health education holds consequences, such as the underrecognition and undertreatment of major depression during pregnancy [1]. Currently, only 59\% of psychiatry programs require any level of training in reproductive psychiatry, and only $36 \%$ of program directors believe that residents need to be competent in the field [2]. Given that 1 in 8 women experience an episode of major depression during pregnancy [1], it is critical that psychiatry residents learn to diagnose and manage women-specific ailments.

The conference and its organizers seek to address this need for education and training. To that end, the trainee-led committee has produced an ambitious agenda for the future. 
To improve education in women's mental health, the committee helped revamp Yale's women's mental health curriculum for the psychiatry residency training program in 2019. The curriculum adapted some modules from the newly established National Curriculum in Reproductive Psychiatry, an online resource that offers education on pressing women's mental health disorders including perinatal mental health, management of gender-based violence, premenstrual dysphoric disorder, and more. To provide clinical education, organizers are actively seeking to partner with local health organizations to create a women's mental health clinic that can provide co-located obstetrics and gynecology and psychiatric care for patients while offering Yale residents a training opportunity in women's mental health.

To highlight the work of existing women's mental health researchers, advocates, clinicians, and patients, the committee is launching a social media campaign to center underrecognized stories and individuals in the field. The committee is also fundraising for local non-profit organizations that provide support for women who experience intimate partner violence, especially during this time of a global pandemic.

Aside from these valuable projects, the committee is also hard at work planning the next iteration of the conference. Due to COVID-19, the event was postponed to Friday, April 23, 2021. By forging an affordable, accessible space where a diversity of professionals can learn together, the conference can spark a collective movement toward a more womanresponsive field of psychiatry.

The success of the inaugural Women's Mental Health Conference and the unwavering commitment of its trainee organizers have been a truly unique experience. No longer are students and trainees talking of improving awareness of women's mental health; rather, they have turned to concrete, progressive action.

\section{Compliance with Ethical Standards}

Disclosure On behalf of all authors, the corresponding author states that there is no conflict of interest.

\section{References}

1. Battle CL, Salisbury AL, Schofield CA, et al. Perinatal antidepressant use: understanding women's preferences and concerns. J Psychiatry Practice. 2013;19:443-53.

2. Osborne L, MacLean JV, Barzilay EM, et al. Reproductive psychiatry residency training: a survey of psychiatric residency program directors. Acad Psychiatry. 2018;42(2):197-201.

Publisher's Note Springer Nature remains neutral with regard to jurisdictional claims in published maps and institutional affiliations. 